\title{
How do dyspnoea scales compare with measurement of functional capacity in patients with COPD and at risk of COPD?
}

\section{*Lonneke M Boer, ${ }^{\mathrm{a},}$, Guus M Asijee ${ }^{\mathrm{c}, \mathrm{d}}$, Onno CP van Schayck ${ }^{\mathrm{d}}$, Tjard RJ Schermer}

\author{
a Department of Medical Psychology, Radboud University Nijmegen Medical Centre, Nijmegen, The Netherlands \\ b Department of Pulmonary Diseases, Radboud University Nijmegen Medical Centre, Nijmegen, The Netherlands \\ Boehringer Ingelheim b.v., Alkmaar, The Netherlands \\ ' CAPHRI School for Public Health and Primary Care, Maastricht University, Maastricht, The Netherlands \\ e Department of Primary and Community Care, Radboud University Nijmegen Medical Centre, Nijmegen, The Netherlands
}

Received 24th November 2011; revised 1st February 2012; accepted 21st February 2012; online 28th March 2012

\begin{abstract}
Background: In primary care, formal functional capacity testing is not always feasible. Guidelines for family practitioners suggest the use of dyspnoea scales to assess exercise tolerance in patients with chronic obstructive pulmonary disease (COPD).

Aims: To examine whether the use of activity-based dyspnoea scales can substitute for actual functional capacity testing.

Methods: 128 subjects (49\% at risk of COPD, 24\% GOLD stage I, 17\% GOLD stage II, 9\% GOLD stage III) performed an Incremental Shuttle Walk Test (ISWT) and completed the Medical Research Council dyspnoea scale (MRC), Baseline Dyspnoea Index (BDI), Oxygen Cost Diagram (OCD), Clinical COPD Questionnaire (CCQ), and St George's Respiratory Questionnaire (SGRQ).

Results: Analysis of variance showed that the relationship between the ISWT and the MRC dyspnoea scale was statistically significant but moderate $\left(p<0.001, R^{2}=0.166\right)$. Correlations between the ISWT and the other dyspnoea scales were also moderate (correlation coefficients $0.34-0.42)$. Combining the dyspnoea scales in one analysis resulted in a proportion of explained variance of the ISWT of $21.4 \%\left(R^{2}=0.214\right)$. Conclusions: Dyspnoea scales cannot substitute for formal functional capacity testing. Authors of COPD guidelines should consider stating more specifically that the MRC and similar scales measure (self-reported) activity-related dyspnoea but cannot replace objectively measured functional capacity.

(C) 2012 Primary Care Respiratory Society UK. All rights reserved.

LM Boer et al. Prim Care Respir J 2012; 21(2): 202-207

http://dx.doi.org/10.4104/pcrj.2012.00031
\end{abstract}

Keyw ords chronic obstructive pulmonary disease, functional capacity, dyspnoea scales, quantitative research

\section{Introduction}

In line with the definition of the Global Initiative for Chronic Obstructive Lung Disease (GOLD) committee, ${ }^{1}$ the severity of COPD is conventionally expressed by the degree of airflow obstruction as measured by spirometry. However, several studies in the past two decades have shown little association between airflow obstruction and other markers of disease severity such as respiratory symptoms, disability, exercise tolerance/physical functional capacity, exacerbations, and health-related quality of life. ${ }^{2,3}$

In response to these findings, additional measures of disease severity have been proposed. ${ }^{2,45} \mathrm{~A}$ growing body of research shows that limitation of functional capacity is a better predictor of disability and mortality in COPD patients than airflow limitation. ${ }^{6.9} \mathrm{Apart}$ from its prognostic value, functional capacity testing is useful in evaluating the effect of therapeutic interventions, and improvement of functional capacity is one of the main treatment goals in the management of COPD. These factors have led to a general consensus on the importance of measuring functional capacity in all patients with COPD. ${ }^{10}$

However, measuring functional capacity requires standardised testing conditions. In a hospital or laboratory setting these requirements are relatively easy to realise, but it is usually difficult to implement standardised functional capacity testing in routine primary care. ${ }^{11}$ National COPD guidelines recommend annual evaluation of exercise limitations or

* Corresponding author: Ms Lonneke Boer, Department of Medical Psychology, Radboud University Nijmegen Medical Centre, Geert Grooteplein Zuid 10, 6525 GA, Nijmegen, The Netherlands. Tel: 0031246859557 Fax: 0031246859531 E-mail: L.Boer@mps.umcn.nl. 
functional capacity and mention the use of dyspnoea scales (especially the Medical Research Council (MRC) scale ${ }^{12}$ ) for performing these measurements. ${ }^{13-15}$ This suggests that exercise tolerance and daily functioning can be measured by a dyspnoea scale. For instance, the MRC score is used to differentiate between patients referred for physical therapy in primary care. ${ }^{15}$ There is, however, little evidence to support the use of dyspnoea scales to assess functional capacity in primary care patients. ${ }^{16}$

In Dutch primary care the majority of patients $(\sim 80 \%)$ have mild to moderate COPD (GOLD stages I and II), ${ }_{17}^{17}$ while most research on functional capacity and dyspnoea scales has been performed in patients with severe to very severe COPD. For the current paper our population consisted mainly of patients with mild to moderate disease and of subjects at risk of developing the disease in years to come (i.e. middle-aged men and women who are current smokers and have chronic cough, sputum production, or dyspnoea). These groups make up the majority of the COPD patient population managed in family practice.

The aim of this study was to examine whether assessment of activity-based dyspnoea - using existing and validated scales can be a useful substitute for the measurement of the actual outcome of interest in COPD (i.e. limitation of functional capacity). Addressing this question is especially important for primary care professionals who are involved in the management of patients with COPD and who do not have access or facilities to perform standardised functional capacity tests.

\section{Methods}

\section{Subjects}

Our study population consisted of 128 individuals (57 men) recruited through newspaper advertisements and radio announcements for a primary care smoking cessation intervention study between January 2001 and November 2001. ${ }^{18}$ Thus, all participants were current smokers. Exclusion criteria were smoking history $<5$ years; history of asthma; acute exacerbation or changes in respiratory treatment regimen in the preceding 4 weeks; and concomitant co-morbidity which may influence functional capacity (i.e. cardiovascular, neurological, endocrine diseases, and/or locomotor limitations). Subjects at risk of COPD were included in this study as a reference group because family practitioners are increasingly encouraged to offer unsolicited advice to patients who smoke, but COPD researchers typically exclude these subjects.

Ethics approval

The Medical Ethics Committee of the Radboud University Nijmegen Medical Centre, Nijmegen, The Netherlands approved the study protocol. All subjects were informed about the study protocol and signed a consent form.

\section{Measurements}

\section{Pulmonary function tests}

Spirometry was performed before and $20 \mathrm{~min}$ after inhalation of two puffs of $200 \mu \mathrm{g}$ salbutamol using a metered-dose inhaler with a spacer device (Volumatic ${ }^{\oplus}$, GlaxoSmithKline, Zeist, The
Netherlands). Inhalation of any bronchodilators was withheld for at least 12 hrs before pulmonary function was tested. Flowvolume curves, forced expiratory volume in $1 \mathrm{~s}\left(\mathrm{FEV}_{1}\right)$, and forced vital capacity (FVC) were measured using a flow screen (Masterlab ${ }^{\circledR}$, Jaeger, Wurzburg, Germany). The highest $\mathrm{FEV}_{1}$ and FVC values of $\geq 3$ acceptable forced manoeuvres were used for analysis. The $\mathrm{FEV}_{1}$ value obtained was related to a reference value and expressed as percentage of the predicted value $\left(\mathrm{FEV}_{1} \%\right) .{ }^{19} \mathrm{Age}$, height $(\mathrm{m})$, weight $(\mathrm{kg})$, and body mass index $\left(\mathrm{BMI}, \mathrm{kg} / \mathrm{m}^{2}\right.$ ) were assessed in each participant.

\section{Assessment of functional capacity}

The Incremental Shuttle Walk Test (ISWT) was used to establish the functional capacity of the study subjects. The ISWT is a standardised test in which subjects have to walk up and down a $10 \mathrm{~m}$ course marked with shuttles. Walking speed is dictated by an audiotape and increases every minute. We adhered to the measurement protocol described by Singh and colleagues..$^{20}$ The outcome measure of the ISWT is the distance walked in metres. The maximum possible score is $1020 \mathrm{~m}$.

\section{Assessment of activity-based dyspnoea}

Activity-based dyspnoea was assessed with five validated dyspnoea scales. The M RC dyspnoea scale assesses any limitation or lack of ability to perform an activity in the manner or within the range considered normal for a human being..$^{12,21}$ The M RC is a single-score scale with a range from 1 ('I only get breathless with strenuous exercise') to 5 ('I am too breathless to leave the house'). In the current study the MRC scale had our specific interest because its use is recommended in several COPD guidelines. ${ }^{13-15,22}$

The Baseline Dyspnoea Index (BDI) assesses three components that evoke dyspnoea: functional impairment, magnitude of task, and magnitude of effort. ${ }^{23}$ The scores of the components are added; the total score ranges from 0 to 12 . Higher scores indicate more dyspnoea.

The Oxygen Cost Diagram (OCD) consists of a list of several daily activities positioned alongside a $100 \mathrm{~mm}$ vertical scale in proportion to their oxygen cost. ${ }^{24}$ Higher scores indicate fewer limitations due to dyspnoea.

The Clinical COPD Questionnaire (CCQ) was developed to measure functional status and symptoms in daily clinical practice. ${ }^{25}$ It consists of three subscales: Symptoms, Functional Status, and Mental State. Item and subscale scores range from 0 (best) to 6 (worst). In the current study only the Functional Status subscale was used.

The St George's Respiratory Questionnaire (SGRQ) measures disease-specific health status and has three subscales: Symptoms, Activity, and Impact. The weighted individual item scores are added and divided by their total subscore. Score range is $0-100$, higher scores indicating more limitations. ${ }^{26}$ In this study only the Activity subscale was used.

Statistical analysis

In this cross-sectional study, statistical analyses were performed using SPSS 16.0 statistical software. 
Figure 2. Mean values of ISWT per MRC score. Bars indicate $95 \%$ confidence intervals. ISWT=Incremental Shuttle Walk Test in metres; MRC=Medical Research Council dyspnoea questionnaire

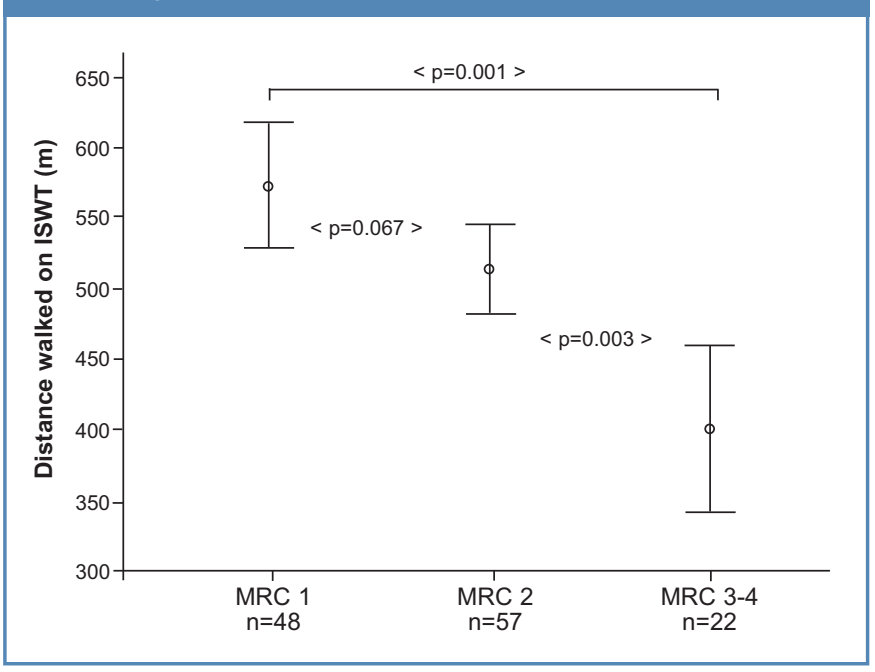

Table 3. Pearson's correlation coefficients between ISWT, BDI, OCD, CCQ Functional Status, and SGRQ Activity

\begin{tabular}{llllll} 
& ISWT & BDI & OCD & $\begin{array}{l}\text { CCQ } \\
\text { Functional } \\
\text { Status }\end{array}$ & $\begin{array}{l}\text { SGRQ } \\
\text { Activity }\end{array}$ \\
\hline ISWT & 1 & & & \\
\hline BDI & $-0.368^{*}$ & 1 & & & \\
\hline OCD & $0.342^{*}$ & $-0.569^{*}$ & 1 & & \\
\hline CCQ-functional & $-0.368^{*}$ & $0.651^{*}$ & $-0.516^{*}$ & 1 & \\
\hline SGRQ-activity & $-0.421^{*}$ & $0.596^{*}$ & $-0.591^{*}$ & $0.628^{*}$ & 1 \\
\hline For abbreviations, see footnote to Table 2. ${ }^{*} \mathrm{p}<0.01$. &
\end{tabular}

significance between MRC scores 2 and $1(p=0.067)$.

Pearson's correlation coefficients between the ISWT, BDI, OCD, CCQ Functional Status and SGRQ Activity were calculated. Correlations between ISWT and the dyspnoea scales were moderate and varied between -0.421 and 0.342 (Table 3).

Combined dyspnoea scales

As a final step in our analyses we performed a univariate ANOVA to examine whether a combined set of dyspnoea scales would explain more of the variance in the ISWT distance than the M RC dyspnoea scale alone. The MRC scale was used as an independent variable and the other dyspnoea scales were added as covariates to the model. This full model explained $22.8 \%$ of the variance in the observed ISWT distances $\left(R^{2}=0.228\right)$. In subsequent steps the OCD, CCQ Functional Status and BDI were excluded from the model as they had $p$ values $>0.10$. This resulted in a reduced model that contained the M RC and SGRQ Activity, both significantly related to the ISWT $(p=0.046$ and $p=0.007$, respectively). This final model explained $21.4 \%$ of the variance in the ISWT observations $\left(R^{2}=0.214\right)$, which is an additional $4.8 \%$ to the $16.6 \%$ of variance explained by the MRC alone.

\section{Subgroup analysis of patients with COPD}

Exploratory analyses were performed on the 64 patients with COPD to examine whether the results would differ if the subjects at risk were excluded. Pearson's correlation coefficients between the ISWT and BDI, OCD, CCQ Functional Status and SGRQ Activity showed a similar patern as the correlations reported in Table $3(-0.462,0.333,-0.355$ and -0.521 , respectively). The M RC dyspnoea scale could explain $21.9 \%$ of the variance of the ISWT. Again, patients with MRC score 3-4 walked significantly shorter distances than patients with MRC scores 1 and 2 $(p<0.001$ and $p=0.02$, respectively), whereas there was no difference in the distance walked between those with MRC scores 1 and $2(p=0.172)$.

Repeating the univariate ANOVA with the MRC and SGRQ Activity, a total of $29.4 \%$ of variance could be explained.

\section{Discussion}

\section{Main findings}

The aim of this study was to examine whether inquiring about dyspnoea-related functional limitations - using existing and validated scales - can substitute for actual measurement of limitation of functional capacity due to dyspnoea in patients with or at risk of COPD.

The commonly used MRC dyspnoea scale showed a statistically significant but only moderate association with the actual functional capacity test. ISWT scores were highest for subjects with MRC score 1 and lowest in subjects with MRC score 3-4, but this difference failed to reach statistical significance between MRC scores 1 and 2 . The relationships between functional capacity and BDI, OCD, CCQ Functional Status subscale, and SGRQ Activity subscale were also moderate. Combining dyspnoea scales in one analysis resulted in a somewhat higher - but still modest - rate of explained variance of the observed ISWT distances. Since the SGRQ Activity subscale has to be weighed and calculated, in our view the extra effort needed if the MRC and SGRQ Activity subscale are combined does not outweigh the limited additional predictive value of the overall instrument.

Interpretation of findings in comparison with previously published work

The moderate relationship between functional capacity and activity-based dyspnoea is not surprising. In the literature, dyspnoea perception and functional capacity are generally viewed as related but separate concepts. ${ }^{27-30}$ More surprising perhaps is that, apparently without a solid scientific base, guidelines for family practitioners suggest that functional capacity can be assessed with an activity-based dyspnoea questionnaire such as the MRC..$^{13-15}$

Health care focuses increasingly on prevention and early detection of COPD, ${ }^{1,13}$ while in most research the pre-clinical stage of COPD is neglected. By including subjects at risk of 
COPD next to subjects with mild to severe airflow obstruction, we attempted to shed some light on the use of activity-based dyspnoea scales as an indicator of limitations in functional capacity in a primary care setting.

In a study by Taylor et al., ${ }^{31}$ mean scores of healthy subjects on the ISWT are reported. After excluding the subjects aged $<30$ for comparison with our population, the mean distance walked by healthy subjects was $646 \mathrm{~m}$. The subjects in our 'at risk' group had a mean ISWT score of $561 \mathrm{~m}$. In a study of the minimal clinically important difference of the ISWT, Singh et al. ${ }^{32}$ found an improvement of $47.5 \mathrm{~m}$ to be 'slightly better' and an improvement of $78.7 \mathrm{~m}$ to be 'better'. The difference of $85 \mathrm{~m}$ between the healthy subjects in the study by Taylor et al. and the subjects at risk in our study could point to a relevant decline of functional capacity in subjects at risk of COPD, a population that is generally excluded from research. Strengths and limitations of this study

In this study the distribution of subjects at risk of COPD and patients with mild, moderate and severe disease was unequal. Half of the subjects were not diagnosed with COPD, which could have a profound impact on the results. We therefore repeated all the analyses without the subjects at risk to check this and found similar results. The proportion of explained variance did increase, but not substantially. In addition, the distances walked by smokers at risk of COPD and the patients with GOLD stage I COPD were very similar (561 m vs. $536 \mathrm{~m}$; Figure 1). We therefore have reason to believe that subjects at risk of COPD are not a distinct population from COPD patients in terms of functional capacity. Furthermore, a study on quality of life in a comparable population also found no difference in the results after excluding people at risk of COPD. ${ }^{33}$

Implications for future research, policy and practice In this study the association between dyspnoea scales and functional capacity was moderate. The MRC and the SGRQ Activity subscale showed the best results, but the effect sizes were moderate. Furthermore, the difference in the mean ISWT walking distance between M RC scores 1 and 2 was not statistically significant. An important observation for daily clinical practice was the wide spread in the distance walked within the MRC scores. For instance, the minimum distance walked by subjects with M RC score 1 ( $290 \mathrm{~m}$ ) was well below the average distance walked by subjects with MRC score 3-4 $(400 \mathrm{~m})$, Thus, although for research purposes the MRC or other dyspnoea scales may have sufficient predictive value, in individual patient care this does not seem to be the case. Healthcare professionals should therefore be cautious in deriving conclusions regarding functional capacity based on dyspnoea scales only.

Functional capacity has been shown to influence the course of COPD, and early detection of decline is important. ${ }^{1,13}$ Approximately $85 \%$ of all COPD patients in the UK and the Netherlands are treated by their family practitioner ${ }^{10,34}$ where standardised functional capacity testing is often not feasible. In that case, referral to a clinic or laboratory with the necessary facilities for testing functional capacity should be considered. Future research on normative and reference data of dyspnoea scales could provide guidelines on when functional capacity testing should be performed (for instance, in patients with MRC score 2), and whether people at risk of COPD could benefit from early detection of limitations in functional capacity.

\section{Conclusions}

Activity-based dyspnoea scales cannot be used as a substitute for actual functional capacity tests in a primary care setting. More dyspnoea may indicate lower functional capacity in patients with COPD, but an actual functional capacity test is still needed to confirm such a finding.

We recommend that COPD guidelines state more specifically that the MRC and other dyspnoea scales measure (self-reported) activity-related dyspnoea but cannot replace objectively measured functional capacity.

\section{Handling editor Niels Chavannes Statistical review Gopal Netuveli}

Acknowledgements The authors would like to thank all the subjects who participated in this study for their time and co-operation; Edwin Wagena, Annie Hendriks, Tom de Rooij, Bart van de Ven (Maastricht University), and Marco Akkermans (Asthma Centre Hornerheide) for organising the measurements and collecting and processing the data; and Boehringer Ingelheim b.v. for financial support.

Conflicts of interest $L M B$ has no conflicts of interest in relation to this article. GMA is an employee of Boehringer Ingelheim b.v. OCPvS is a consultant for Boehringer Ingelheim b.v., AstraZeneca and Pfizer. TRJS is a consultant for Boehringer Ingelheim b.v., GSK and AstraZeneca. OCPvS is an Assistant Editor of, and TRJS an Associate Editor of, the PCRJ; neither were involved in the editorial review of, nor the decision to publish, this article.

Contributorship Study concept and design, analysis and interpretation of data, and critical revision of the manuscript for important intellectual content: LMB, GMA, OCPVS, TRJS. Acquisition of data: OCPVS, TRJS. Drafting of the manuscript: LM B under the supervision of TRJS. Statistical analysis: LM B, TRJS. Obtained funding: TRJS. Study supervision: TRJS.

Funding This work was supported by Boehringer Ingelheim b.v., The Netherlands.

\section{References}

1. Global strategy for the Diagnosis, Management, and Prevention of COPD, Global Initiative for Chronic Obstructive Lung Disease (GOLD) 2010. http://www.goldcopd.org/ (accessed 10 Nov 2011).

2. Celli BR, Cote CG, Lareau SC, Meek PM. Predictors of survival in COPD: more than just the FEV $\mathrm{F}_{1}$. Respir M ed 2008;102(Suppl 1):S27-35. http://dx.doi.org/10.1016/ S0954-6111(08)70005-2

3. Curtis JR, Martin DP, Martin TR. Patient-assessed health outcomes in chronic lung disease: what are they, how do they help us, and where do we go from here? Am J Respir Crit Care Med 1997;156:1032-9.

http://ajrccm.atsjournals.org/cgi/ content/full/156/4/1032

4. Cazzola M, Hanania NA, Jones PW, et al. It's about time - directing our attention toward modifying the course of COPD. Respir Med 2008;102(Suppl 1):S37-48. http://dx.doi.org/10.1016/S0954-6111(08)70006-4

5. Oga T, Nishimura K, Tsukino M, Sato $S$, Hajiro T. Analysis of the factors related to mortality in chronic obstructive pulmonary disease: role of exercise capacity and health status. Am J Respir Crit Care Med 2003;167(4):544-9. http://dx.doi.org/10.1164/rccm.200206-5830C

6. Casanova C, Cote C, M arin JM, et al. Distance and oxygen desaturation during the 
6-min walk test as predictors of long-term mortality in patients with COPD. Chest 2008;134(4):746-52. http://dx.doi.org/10.1378/chest.08-0520

7. Celli BR. Predictors of mortality in COPD. Respir Med 2010;104(6):773-9. http://dx.doi.org/10.1016/j.rmed.2009.12.017

8. Dolan S, Varkey B. Prognostic factors in chronic obstructive pulmonary disease. Curr Opin Pulm Med 2005;11(2):149-52. http://dx.doi.org/10.1097/ 01.mcp.0000153548.36054.8f

9. Spruit MA, Polkey MI, Celli B, et al. Predicting outcomes from 6-minute walk distance in chronic obstructive pulmonary disease. J Am Med Dir Assoc 2012;13(3):291-7. http://dx.doi.org/10.1016\%2Fj.jamda.2011.06.009

10. Palange $\mathrm{P}, \mathrm{Ward} \mathrm{SA}, \mathrm{Carlsen} \mathrm{KH}$, et al. Recommendations on the use of exercise testing in clinical practice. Eur Respir J 2007;29(1):185-209. http://dx.doi.org/10.1183/09031936.00046906

11. Haughney J, Gruffydd-Jones K. Patient-centred outcomes in primary care management of COPD - what do recent clinical trial data tell us? Prim Care Respir J 2004;13(4):185-97. http://dx.doi.org/10.1016/j.pcrj.2004.06.006

12. Fletcher $\mathrm{CM}$, Elmes $\mathrm{PC}$, Fairbairn $\mathrm{AS}$, Wood $\mathrm{CH}$. The significance of respiratory symptoms and the diagnosis of chronic bronchitis in a working population. Br Med J 1959;2:257-66. http://dx.doi.org/10.1136/bmj.2.5147.257

13. National Clinical Guideline Centre. Chronic obstructive pulmonary disease: management of chronic obstructive pulmonary disease in adults in primary and secondary care. London: National Clinical Guideline Centre, 2010. http://guidance.nice.org.uk/ (accessed 10 Nov 2011).

14. O'Donnell DE, Aaron S, Bourbeau J, et al. Canadian Thoracic Society recommendations for management of chronic obstructive pulmonary disease - 2007 update. Can Respir J 2007;14(Suppl B):5B-32B. http://www.ncbi.nlm.nih.gov/pmc/ articles/PM C2806792/

15. Smeele IJM, van Weel C, van Schayck CP, et al. NHG-Standaard COPD. Second Revision. Huisarts Wet 2007; 50:362-79.

16. Wegner RE, Jorres RA, Kirsten DK, Magnussen $\mathrm{H}$. Factor analysis of exercise capacity, dyspnoea ratings and lung function in patients with severe COPD. Eur Respir J 1994;7(4):725-9. http://dx.doi.org/10.1183/09031936.94.07040725

17. Hoogendoorn M, Rutten-van Molken MP, Hoogenveen RT, et al. A dynamic population model of disease progression in COPD. Eur Respir J 2005;26(2):223-33. http://dx.doi.org/10.1183/09031936.05.00122004

18. Wagena EJ, Knipschild PG, Huibers MJ, Wouters EF, van Schayck CP. Efficacy of bupropion and nortriptyline for smoking cessation among people at risk for or with chronic obstructive pulmonary disease. Arch Intern Med 2005;165(19):2286-92. http://dx.doi.org/10.1001/archinte.165.19.2286

19. Quanjer PH, Tammeling GJ, Cotes JE, Pedersen OF, Peslin R, Yernault JC. Lung volumes and forced ventilatory flows. Report Working Party Standardization of Lung Function Tests, European Community for Steel and Coal. Official Statement of the European Respiratory Society. Eur Respir J Suppl 1993;16:5-40.

20. Singh SJ, Morgan MD, Scott S, Walters D, Hardman AE. Development of a shuttle walking test of disability in patients with chronic airways obstruction. Thorax 1992;47(12):1019-24. http://dx.doi.org/10.1136/thx.47.12.1019
21. Bestall JC, Paul EA, Garrod R, Garnham R, Jones PW, Wedzicha JA. Usefulness of the Medical Research Council (M RC) dyspnoea scale as a measure of disability in patients with chronic obstructive pulmonary disease. Thorax 1999;54(7):581-6. http://dx.doi.org/10.1136/thx.54.7.581

22. McKenzie DK, Abramson M, Crockett AJ, et al. on behalf of The Australian Lung Foundation. The COPD-X Plan: Australian and New Zealand. Guidelines for the management of chronic obstructive pulmonary disease 2011. http://www.copdx.org.au/ (accessed 22 Dec 2011).

23. Mahler DA, Weinberg DH, Wells CK, Feinstein AR. The measurement of dyspnea. Contents, interobserver agreement, and physiologic correlates of two new clinical indexes. Chest 1984;85(6):751-8. http://dx.doi.org/10.1378/chest.85.6.751

24. McGavin CR, Artvinli M, Naoe H, McHardy GJ. Dyspnoea, disability, and distance walked: comparison of estimates of exercise performance in respiratory disease. $\mathrm{Br}$ Med J 1978;2:241-3. http://dx.doi.org/10.1136/bmj.2.6132.241

25. Van Der Molen T, Willemse BW, Schokker S, Ten Hacken NH, Postma DS, Juniper EF. Development, validity and responsiveness of the Clinical COPD Questionnaire. Health Qual Life Outcomes 2003;1(1):13. http://dx.doi.org/10.1186/1477-7525-1-13

26. Jones PW, Quirk FH, Baveystock CM, Littlejohns P. A self-complete measure of health status for chronic airflow limitation. The St George's Respiratory Questionnaire. Am Rev Respir Dis 1992;145(6):1321-7.

27. Celli BR, Cote CG, Marin JM, et al. The body-mass index, airflow obstruction, dyspnea, and exercise capacity index in chronic obstructive pulmonary disease. $\mathrm{N}$ Engl J Med 2004;350(10):1005-12. http://dx.doi.org/10.1056/NEJM oa021322

28. Foglio K, Carone M, Pagani M, Bianchi L, Jones PW, Ambrosino N. Physiological and symptom determinants of exercise performance in patients with chronic airway obstruction. Respir Med 2000;94(3):256-63. http://dx.doi.org/10.1053/ rmed.1999.0734

29. Kocks JW, Asijee GM, Tsiligianni IG, Kerstjens HA, van der M olen T. Functional status measurement in COPD: a review of available methods and their feasibility in primary care. Prim Care Respir J 2011;20(3):269-75. http://dx.doi.org/10.4104/ pcrj.2011.00031

30. Vercoulen JH, Daudey L, Molema J, et al. An Integral assessment framework of health status in chronic obstructive pulmonary disease (COPD). Int J Behav Med 2008;15(4):263-79. http://dx.doi.org/10.1080/10705500802365474

31. Taylor S, Frost H, Taylor A, Barker K. Reliability and responsiveness of the shuttle walking test in patients with chronic low back pain. Physiother Res Int 2001;6(3):170-8. http://dx.doi.org/10.1002/pri.225

32. Singh SJ, Jones PW, Evans R, Morgan MD. Minimum clinically important improvement for the incremental shuttle walking test. Thorax 2008;63(9):775-7. http://dx.doi.org/10.1136/thx.2007.081208

33. Geijer RM, Sachs AP, Verheij TJ, Kerstjens HA, Kuyvenhoven M M, Hoes AW. Quality of life in smokers: focus on functional limitations rather than on lung function? $\mathrm{Br}$ J Gen Pract 2007;57(539):477-82.

34. Tirimanna PR, van Schayck CP, den Otter JJ, et al. Prevalence of asthma and COPD in general practice in 1992: has it changed since 1977? Br J Gen Pract 1996;46(406):277-81.

\section{Available online at http://ww w.thepcrj.org}

